\title{
Girder deformation related phase errors on the undulators for the European X-Ray Free Electron Laser
}

\author{
Yuhui $\mathrm{Li}^{1,{ }^{1 *}}$ Bora Ketenoglu, ${ }^{1,2}$ and Joachim Pflueger ${ }^{1}$ \\ ${ }^{1}$ European XFEL, Notkestrasse 85, 22607 Hamburg, Germany \\ ${ }^{2}$ Department of Engineering Physics, Ankara University, 06100 Ankara, Turkey
}

(Received 13 April 2015; published 22 June 2015)

\begin{abstract}
In long gap tunable undulators, strong magnetic forces always lead to some amount of gap-dependent girder deformation and resulting gap-dependent phase errors. For the undulators for the European XFEL, this problem has been investigated thoroughly and quantitatively. Using the different gap dependencies of suitable shims and pole height tuning, a method is presented which can be applied to reduce the overall gap dependence of the phase error if needed. It is exemplified by tuning one of the undulator segments for the European X-Ray Free Electron Laser back to specs.
\end{abstract}

DOI: 10.1103/PhysRevSTAB.18.060704

PACS numbers: 41.60.Cr

\section{INTRODUCTION}

The European X-Ray Free Electron Laser (EXFEL) facility is currently under construction in Hamburg, Germany [1]. Its electron beam energy is variable between 8.5 and $17.5 \mathrm{GeV}$. Using the principle of self-amplified spontaneous emission (SASE), [2,3] intense laser radiation is generated in three gap tunable undulator systems called SASE1, SASE2, and SASE3. SASE1 and SASE2 are hard $\mathrm{x}$-ray FELs using 35 undulator segments each with a period length of $40 \mathrm{~mm}$, called U40s. The total length is $205 \mathrm{~m}$. By a suitable choice of beam energy and undulator gap, the wavelength can be tuned from 0.05 to $0.4 \mathrm{~nm}$. SASE3 is a soft x-ray FEL using 21 undulator segments with a period length of $68 \mathrm{~mm}$, called U68s, and a total length of $121 \mathrm{~m}$. Its wavelength can be tuned from 0.4 to $5.2 \mathrm{~nm}$. All undulator segments of the EXFEL are $5 \mathrm{~m}$ long and use identical mechanical drive and support systems, which are designed to comply with worst-case requirements. The EXFEL undulator systems are subdivided into cells. Each comprises a $5 \mathrm{~m}$ long undulator segment and a $1.1 \mathrm{~m}$ long intersection, which carries a quadrupole for beam focusing, a phase shifter to match the microbunched electron beam with the radiation field, a beam position monitor, correctors, and a vacuum pump. Table I gives a summary of specifications for the undulator systems for EXFEL.

A strong magnetic force is acting between the girders of an undulator, which is proportional to the square of magnetic field and therefore strongly gap dependent as well. For example, in an U68 operated at a lowest gap of $10 \mathrm{~mm}$, the

\footnotetext{
*Corresponding author. yuhui.li@xfel.eu

Published by the American Physical Society under the terms of the Creative Commons Attribution 3.0 License. Further distribution of this work must maintain attribution to the author(s) and the published article's title, journal citation, and DOI.
}

maximum magnetic force amounts to about 17 tons. This leads to unavoidable mechanic deformation of the girders, resulting in a modulation of the parallel gap profile. Although it can be minimized by a suitable mechanic design, it cannot be avoided completely. Moreover, for a given girder cross section, deformation increases with the third power of its length. Therefore, the mechanical design of the girders for the $5 \mathrm{~m}$ long undulator segments for the EXFEL needed to be a compromise between acceptable girder deformation and technical effort, i.e., amount of material and cross section. This paper concentrates on the effects of girder deformation, i.e., small variations of the parallelism of the magnetic gap along the undulator, its gap dependence, and the impact on magnetic and optical properties. Mechanical deformation is considered as an unavoidable fact. Its causes are not the subject of this paper. But in order to reduce its effects a compensation method is described which significantly reduces gap-dependent changes of the optical phase resulting from girder deformation. Results are exemplified on an undulator segment of the EXFEL.

\section{PHASE ERRORS INDUCED BY GAP DEFORMATION}

\section{A. Phase jitter, deformation, and undulator parameter}

The peak field of permanent magnet structures depends only on the ratio of the gap to the period length, $g / \lambda_{u}$. For a specific structure, the peak field $B_{0}$ can be described by a modified exponential. A common form, taken from Ref. [4] and used throughout this report, is

$$
B_{0}=a \cdot \exp \left[b\left(\frac{g}{\lambda_{u}}\right)+c\left(\frac{g}{\lambda_{u}}\right)^{2}\right]
$$

Here $a, b$, and $c$ are fitting parameters. They depend on very specific design details of the magnet structure such as 
TABLE I. Specifications for the undulator segments of the EXFEL.

\begin{tabular}{lcc}
\hline \hline & SASE1/2 & SASE3 \\
\hline Undulator type & $\mathrm{U} 40$ & $\mathrm{U} 68$ \\
Period length [mm] & 40 & 68 \\
Segment length [m] & 5 & 5 \\
Total number of poles & 248 & 146 \\
Operational gap range [mm] & $10-20$ & $10-25$ \\
Max. peak field @ 10 mm [T] & 1.11 & 1.66 \\
$K$ parameter range in operational & $1.65-3.9$ & $4-9$ \\
$\quad$ gap range & & \\
Cell length [m] & 6.1 & 6.1 \\
Intersection length [m] & 1.1 & 1.1 \\
Number of cells per system & 35 & 21 \\
System length [m] & 205 & 121 \\
Max. phase jitter [degree] & $\leq 8$ & $\leq 8$ \\
Radiation wavelength [nm] & $0.05-0.4$ & $0.4-5.2$ \\
\hline \hline
\end{tabular}

TABLE II. Fitting results for $a, b$, and $c$ for the U40 and U68 undulators obtained from magnetic measurements in the gap range $10-100 \mathrm{~mm}$. They describe the field with a typical $\mathrm{rms}$ residual error of $\leq 5 \times 10^{-4} \mathrm{~T}$.

\begin{tabular}{lcccc}
\hline \hline & Number of poles & $a$ & $b$ & $c$ \\
\hline U40 & 246 & 2.915 & -4.02 & 0.52 \\
U68 & 144 & 3.214 & -4.62 & 0.93 \\
\hline \hline
\end{tabular}

pole and magnet dimensions, properties of magnet and pole material, etc. Table II reproduces $a, b$, and $c$ obtained from fits to magnetic measurement measurements for the EXFEL U40 and U68 structures. The gap range is 10-100 mm. Note that outside Eq. (1) diverges.

Gap errors $\delta g$ and magnetic field errors $\delta B$ are related by

$$
\delta g=\frac{\delta B}{B_{0}}\left(\frac{b}{\lambda_{u}}+\frac{2 c}{\lambda_{u}^{2}} g\right)^{-1}
$$

The gap and field change have opposite sign.

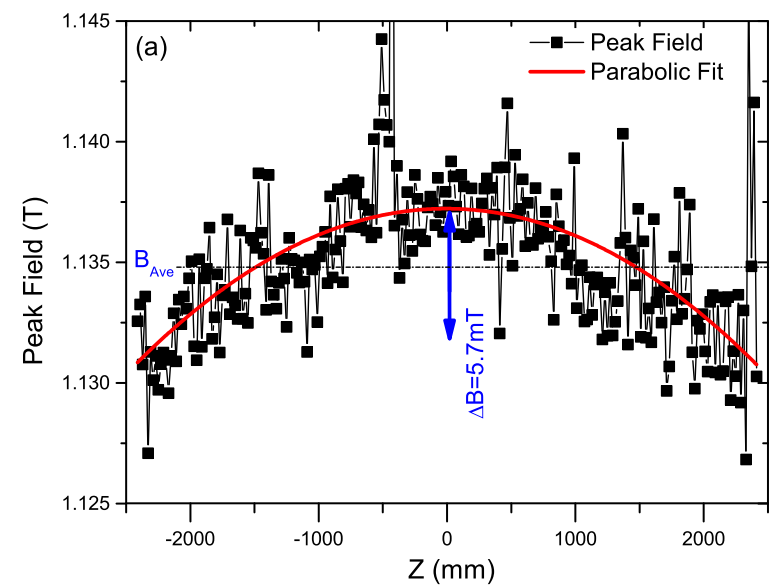

Figure 1(a) shows the variation of the absolute values of the peak fields of the 246 poles of a $5 \mathrm{~m}$ long EXFEL U40 at $10 \mathrm{~mm}$ gap. The origin of the $z$ coordinate is in the center of the structure. The positions of poles extend over $\pm 2500 \mathrm{~mm}$, the full length of the structure. A clear parabolic shape of the peak fields vs $z$ is visible, which can be described by a quadratic parabola of the form

$$
B_{0}(z)=A+C \cdot z^{2}
$$

$A$ and $C$ are empiric constants. A fit to the data points in Fig. 1(a) is shown by the red curve. It is seen that relative to the indicated average there is a variation of about $\pm 2.85 \mathrm{mT}$ or $\pm 0.28 \%$. Because of magnetic forces, the gap in the center is smaller and the peak fields are correspondingly higher. Figure 1(b) shows the corresponding gap variation of the poles using Eq. (2). It is parabolic as well. Because of the forces, the gap varies by $\pm 48 \mu \mathrm{m}$ over the whole length. This behavior is quite typical for all undulator segments of the EXFEL.

The variation of the peak field along the undulator results in a variation of the $K$ parameter. For a sinusoidal field it is given by

$$
K=\frac{e}{2 \pi m c} B_{0} \lambda_{u}=0.09337 \cdot B_{0}[\mathrm{~T}] \cdot \lambda_{u}[\mathrm{~mm}]
$$

so that $\frac{\Delta K}{K}=\frac{\Delta B}{B_{0}}$. The variation of the $K$ parameter along $z$ results in an optical phase jitter $\sigma_{\varphi}$. The effect of different girder deformation profiles on FEL properties was investigated in Ref. [5]. The basic equation for phase jitter is

$$
\sigma_{\varphi}=\alpha \frac{4 \pi}{\lambda_{u}} \frac{1}{1+2 K^{-2}} \frac{\Delta K}{K} \lambda_{\delta}
$$

where $\alpha$ is a coefficient which relates to the geometry and shape of a specific error. $\lambda_{\delta}$ is the characteristic extension

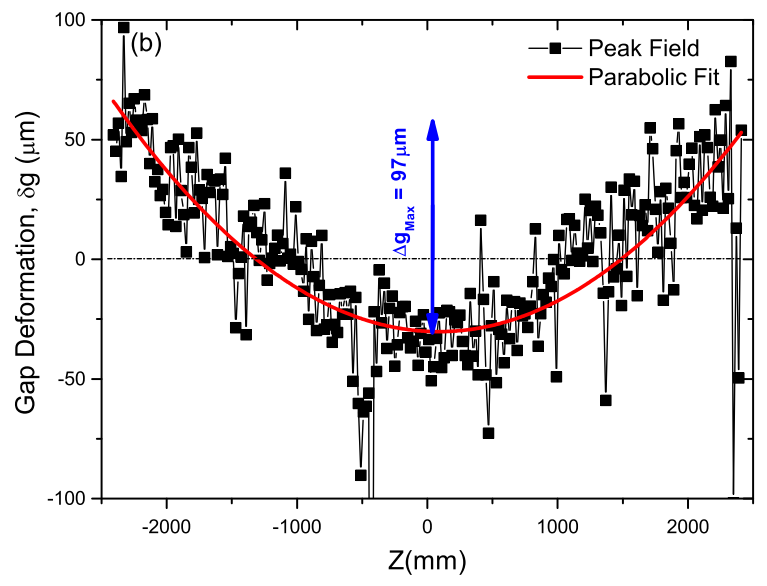

FIG. 1. The measured peak fields (a) and the calculated gap deformation (b). The gap is $10 \mathrm{~mm}$. The red curves show parabola fits; see Eq. (3). The undulator U40-X043 is used to demonstrate the deformation. 


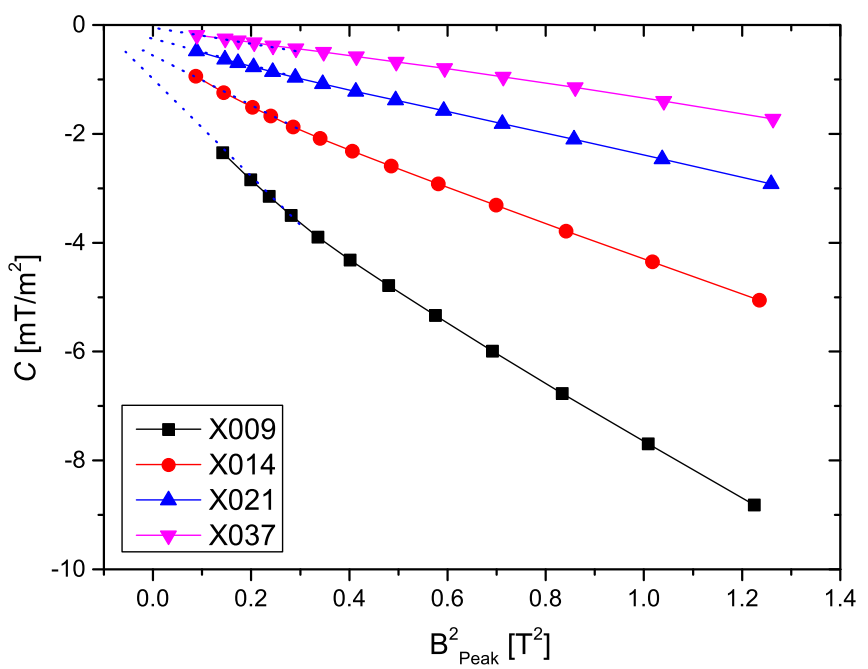

FIG. 2. Coefficient $C$ [see Eq. (3)] vs square of the peak field to demonstrate the variation of girder deformation. The black data points result from initial measurements on a device which was rejected and reconstructed.

length of the error. $\Delta K$ is the total max-min variation of the $K$ parameter.

Parabolic deformation extends homogeneously over the whole undulator, so $\lambda_{\delta}=5 \mathrm{~m}$, the full length of the device. For a given magnet structure defined by $K$ and $\lambda_{u}$ the phase jitter is determined by the product of $\Delta K$ and $\lambda_{\delta}$. So short extension errors can tolerate larger $\Delta K$ and vice versa.

In Ref. [5], $\alpha$ was calculated for periodic parabolic girder deformation, which required periodic solutions for the phase error resulting in $\alpha=3 \sqrt{6 / 35}=1.242$. In contrast in this paper, $K$ variation is nonperiodic and the average $K$ parameter is chosen such that the rms phase jitter is minimized. Following the idea of Ref. [5] and taking this difference as well as a different definition of $\Delta K$ and $\lambda_{\delta}$ into account, $\alpha=\sqrt{1 / 1575}=0.0252$ is obtained.

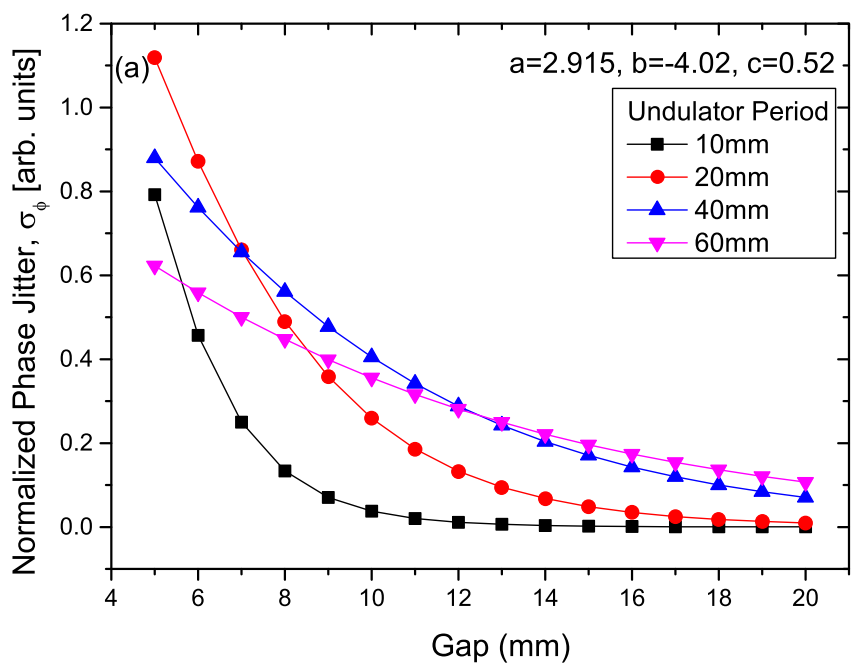

Girder deformation $\Delta g$ is proportional to the magnetic force, which scales with the square of the undulator field, $B_{0}^{2}$. On all EXFEL devices it has been observed that the proportionality constant varies significantly from device to device and can only be determined empirically. The variation is demonstrated in Fig. 2 for four different U40s. The quadratic coefficient $C$ [see Eq. (3)] is plotted as a function of the square of the peak field over the operational gap range. It is seen that there is good overall linear dependence. Only at small $B_{0}^{2}$, where forces are small, are there slight deviations. The slope representing the deformation changes up to a factor of about 5. The black data, however, are quite extreme. They are initial data from a device, which was rejected and completely reconstructed. For all curves, extrapolation to $B_{0}^{2}=0$ shows that there is some small initial girder deformation at large gaps.

According observations were made on all 91 undulator segments, which were built for EXFEL; see also below. The cause of the scatter of the deformation is not fully understood yet and will be subject to more investigations in the future.

Without specific knowledge of the magnitude and type of errors, the combination of Eqs. (1),(2),(4),(5) still allows one to give a proportionality relation for the phase jitter:

$$
\sigma_{\varphi} \propto \frac{1}{\lambda_{u}^{2}} \frac{b+2 c \frac{g}{\lambda_{u}}}{1+2 K^{-2}} B_{0}^{2}
$$

In order to study trends, Figs. 3(a) and 3(b) show graphs of Eq. (6) for gaps from 5 to $20 \mathrm{~mm}$ and $\lambda_{u}$ from 10 to $80 \mathrm{~mm}$. EXFEL U40 type magnet designs are assumed for the constants $a, b$, and $c$; see Table II. Here are some observations: It is seen in Fig. 3(a) that for a specific $\lambda_{u}$ the phase jitter decreases monotonically with increasing gap but there is no monotonic trend for $\lambda_{u}$.

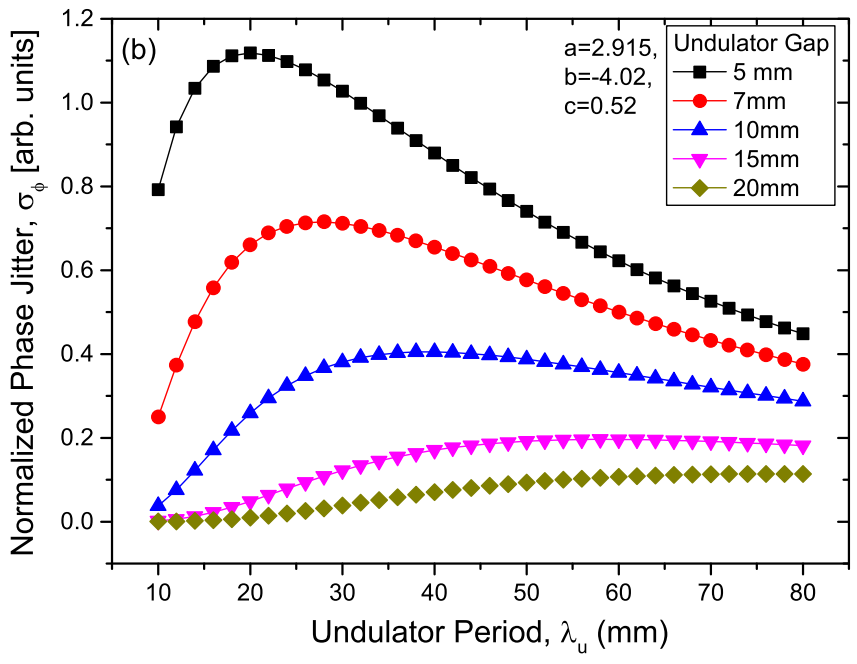

FIG. 3. The normalized phase jitter as a function of the undulator gap (a) and as a function of the undulator period (b). 
Figure 3(b) shows the dependence for five different gaps on the period length. The smaller the gap, the larger the maximum $\sigma_{\varphi}$. In addition, there is a maximum of $\sigma_{\varphi}$ vs $\lambda_{u}$, which is very pronounced at small gaps. For a $5 \mathrm{~mm}$ gap it is at $\lambda_{u} \approx 20 \mathrm{~mm}$ for a $10 \mathrm{~mm}$ gap, the EXFEL gap; it is more shallow and around $\lambda_{u} \approx 35-40 \mathrm{~mm}$. It is seen that the EXFEL U40s with $\lambda_{u}=40 \mathrm{~mm}$ are quite sensible to deformation. In contrast, the sensibility to deformation for the EXFEL U68s with $\lambda_{u}=68 \mathrm{~mm}$ is reduced by about $25 \%$. The larger the gap, the smaller the sensibility to deformation.

\section{B. Gap-dependent parabolic deformation}

On all EXFEL undulators, gap-dependent parabolic deformation is observed to some extent. Pole height tuning (PHT) is used as the standard tool for field error correction, which allows one to shift each pole vertically by about $\pm 300 \mu \mathrm{m}[6,7]$. It is a perfect tool for static corrections of any deformation at one gap. In order to limit overall deformation and its effect on phase jitter, a "tuning gap" was selected, which is about halfway inside the operational gap range. Gaps of 14 and $16 \mathrm{~mm}$ were selected for U40s and U68s, respectively. At the tuning gap any deformation of the poles is completely eliminated by using PHT. The resulting deformation profile of the poles is sketched in Figs. 4(a)-4(c): At the lowest gap, Fig. 4(a), there is only moderate concave deformation of the poles. At the tuning gap, Fig. 4(b), there is none. Above the tuning gaps, the pole deformation gets convex. Two points should be emphasized: (i) Girder deformation is small, as already shown in Fig. 1. Typical pole height adjustments to compensate deformation are in the range $\pm 50-60 \mu \mathrm{m}$ or less. (ii) The focus is on pole deformation. The deformation of the aluminum support girders cannot be changed. They are perfectly flat only under force-free conditions at very large gaps and gradually deform from flat to concave towards small gaps. This situation is sketched in Figs. 4(a)-4(c) as well.

Parabolic gap deformation is quantified by $\Delta g$ and defined by the difference of the gap as measured at the ends minus the gap measured in the center. Accordingly, for concave deformation at small gaps $\Delta g>0$. At the tuning gap $\Delta g=0$ and at large gaps, the convex deformation case $\Delta g<0$.

The result of girder deformation is that the $K$ parameter slightly varies parabolically along the undulator axis by typically a few tenths of a percent as shown in Fig. 1. As a result the phase error varies [5]:

$\delta \varphi(z)=-\frac{4 \pi}{1+2 K^{-2}} \frac{\Delta g}{\lambda_{u}{ }^{2}}\left(b+\frac{2 c g}{\lambda_{u}}\right)\left(\frac{4}{3 L_{U}^{2}} z^{3}-\frac{1}{5} z\right)$.

$L_{U}$ is the undulator length. $\Delta g$ can be positive or negative, and $z$ was defined in Fig. 1. For a pure parabolically shaped deformation like the one shown in Fig. 1, the resulting phase error has odd symmetry with respect to the undulator center.

Figure 5 illustrates the phase error $\delta \varphi$ as a result of parabolic pole deformation of the undulator U40-X014. The pole tuning was done at a gap of $14 \mathrm{~mm}$. Figure 5(a) shows the phase error at a gap of $10 \mathrm{~mm}$, Fig. 5(b) at $20 \mathrm{~mm}$. The black curves are the phase errors obtained from magnetic measurements. The smooth red curves are calculated by using Eq. (7) using $\Delta g$ from measurements such as shown in Fig. 1. Measured and calculated results agree fairly well. It is quite obvious that the dominant source of phase errors is girder deformation. Short-range contributions from magnetic field errors are visible but are much smaller. Between Figs. 5(a) and 5(b), there is a change in the sign of $\Delta g$ which is reflected in the phase error.

The rms phase jitter $\sigma_{\varphi}$ obtained from $\delta \varphi(z)$ can be calculated from Eq. (7); see Ref. [5]:

$$
\sigma_{\varphi}=\sqrt{\frac{16}{1575}} \pi \frac{\left|\frac{b}{\lambda_{u}}+\frac{2 c g}{\lambda_{u}{ }^{2}}\right|}{1+2 K^{-2}} N_{U}|\Delta g| .
$$

$\sigma_{\varphi}$ is proportional to the product of the undulator period number $N_{U}$ and the gap deformation $\Delta g$.

In Fig. 6, the maximum gap deformation $\Delta g$ is plotted as a function of the gap using Eq. (8) for U40 and U68 parameters taken from Table I. The tolerance for the phase jitter, $8^{\circ}$, is a conservative choice and originated in

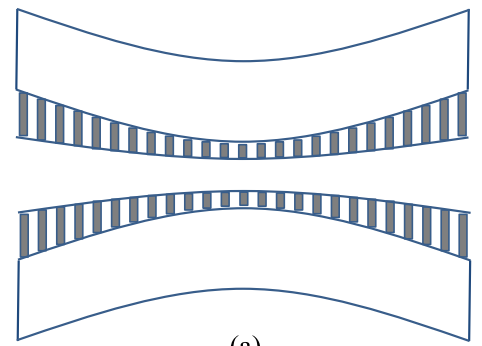

(a)
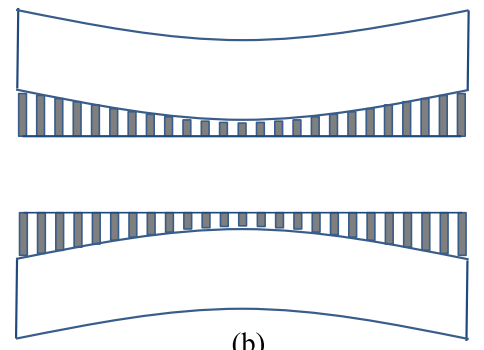

(b)
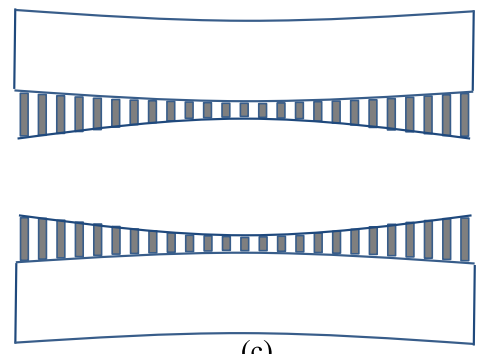

FIG. 4. Girder and pole deformation on the EXFEL undulators with applied pole height tuning. (a) At small gaps $\Delta g>0$, concave case; (b) at the tuning gap $\Delta g=0$, flat case; (c) at large gaps $\Delta g<0$, convex case. The aluminum support girders gradually deform from convex at small gaps to flat at very large gaps. 

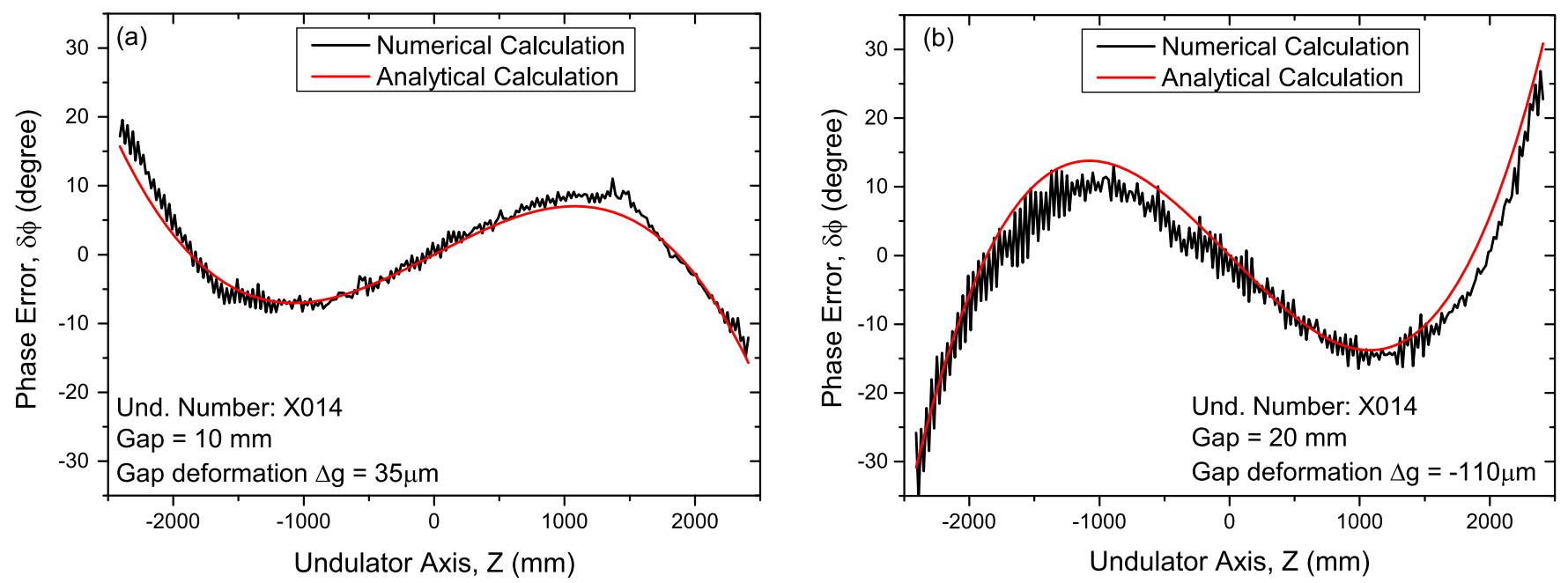

FIG. 5. The phase error induced by gap deformation for the U40-X014. (a) The gap is $10 \mathrm{~mm}$ and the deformation is $35 \mu \mathrm{m}$. (b) The gap is $20 \mathrm{~mm}$ and the deformation is $-110 \mu \mathrm{m}$. The black curves are obtained from magnetic field measurements, the red curves from Eq. (7).

Genesis1.3 studies reported in Ref. [5] which required $10^{\circ}$ for a reduction of FEL power of less than $10 \%$. Nevertheless, $8^{\circ}$ was chosen to have some contingency [8]. The experience showed that it is achievable with reasonable effort.

The curves are symmetric for positive and negative $\Delta g$ corresponding to concave and convex deformation, respectively. Figure 6 defines a specification window. It is seen that the tolerance for gap deformation increases with increasing gap. There is also a difference between the U40 and the U68. For a U40 $\Delta g$ extends from $\pm 50 \mu \mathrm{m}$ at $10 \mathrm{~mm}$ gap to about $\pm 75 \mu \mathrm{m}$ at $20 \mathrm{~mm}$ gap, for a U68 from $\pm 110 \mu \mathrm{m}$ at $10 \mathrm{~mm}$ to $\pm 120 \mu \mathrm{m}$ at $25 \mathrm{~mm}$ gap. This trend is also seen in Fig. 3(b). Note that the deformation at $20 \mathrm{~mm}$ gap given in Fig. 5(b) is

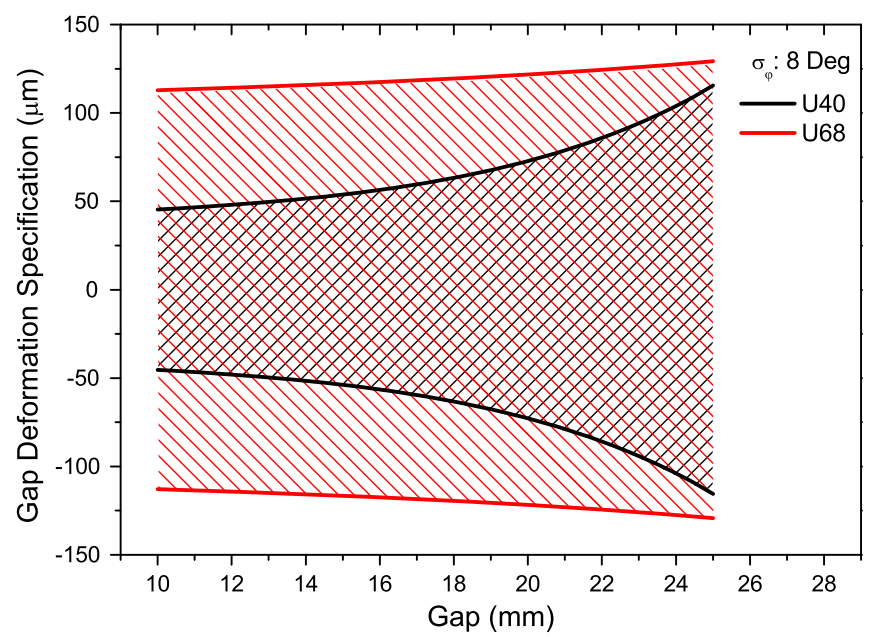

FIG. 6. Specification window for gap deformation for U40 (black) and U68 (red) undulators. The rms phase jitter tolerance is $8^{\circ}$. unacceptably large and gave rise to more investigations, which are described below.

\section{Observed girder deformation on the EXFEL undulators}

Gap-dependent deformation and phase jitter are shown in Fig. 7 for ten representative U40s. Considerable variation between individual devices is observed, which can be grouped in classes.

Hollow symbols (X002, X045 X092, X044) indicate small, half-filled symbols (X043, X055) moderate, and full symbols (X005, X006, X007) large gap dependence. These are all inside the specification window. As mentioned above, there is one, marked with "+", X014, which is outside the specified range.

The gap dependence of the phase jitter is shown in Fig. 7(b) for the same devices. Again, all except X014 are below the tolerance limit of $8^{\circ}$. The low deformation devices show low variation of phase jitter as well. On all devices the phase jitter shows a minimum of around $2^{\circ}$ near $14 \mathrm{~mm}$, which corresponds to the tuning gap. Figure 7(b) is another demonstration that the gapdependent phase jitter observed on the EXFEL undulators is dominated by mechanical deformation.

\section{COMPENSATION OF GAP DEPENDENCE}

Fortunately, the X014 undulator segment is the only one among the 91 undulator segments built for EXFEL, which does not comply with deformation specs.

Although the reason is not fully understood and still under investigation, a time-consuming analysis of the mechanical support system and refurbishment was avoided in favor of a timely completion. Instead, a compensation method based on using shims was developed, which is 

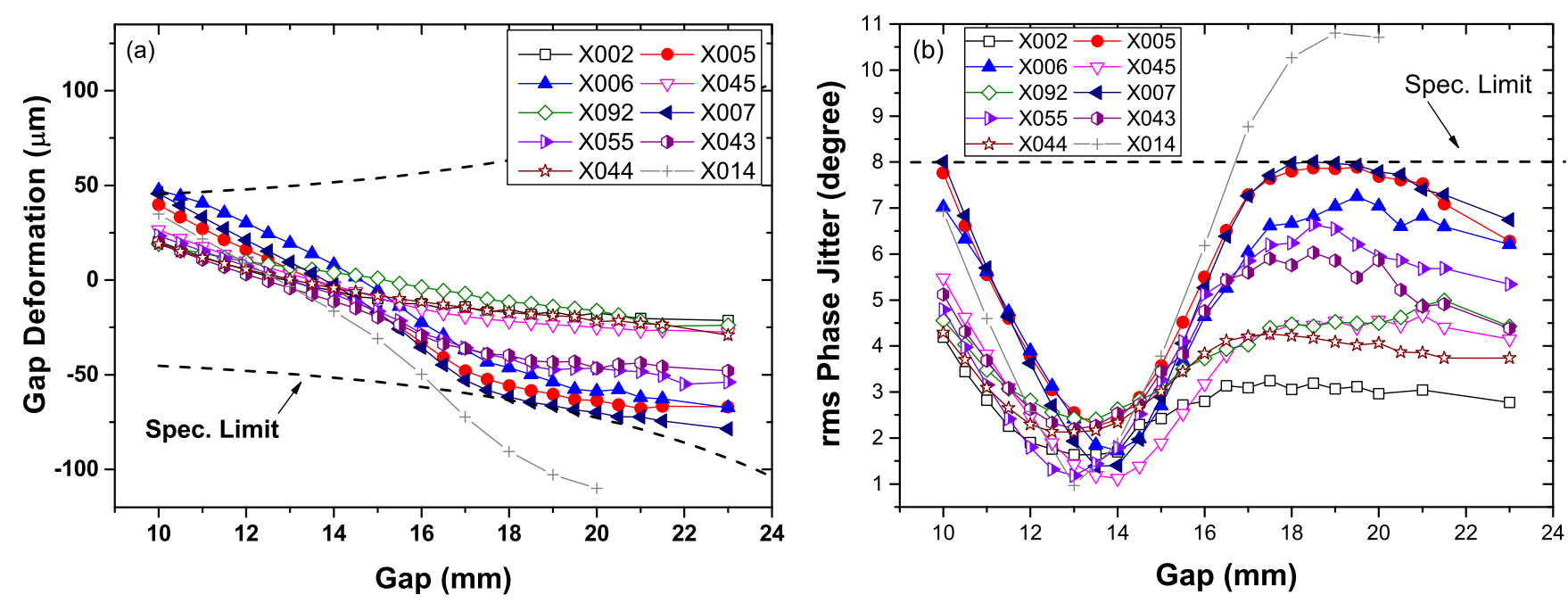

FIG. 7. The status of ten representative U40 undulator segments. (a) Gap dependence of deformation. (b) Gap dependence of rms phase jitter. Specification limits are indicated.

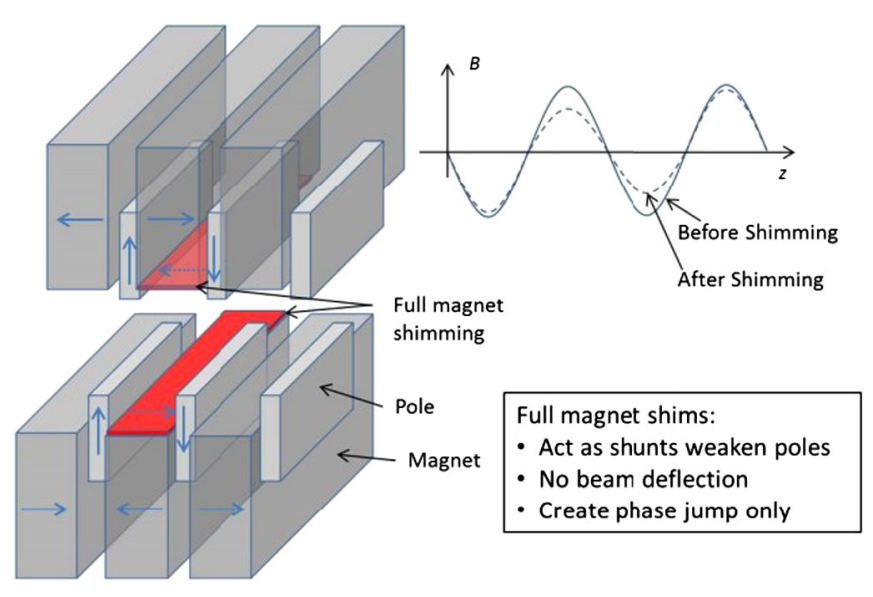

FIG. 8. Principle of full magnet shims.

described below. It is closely related to the shimming method used at the EXFEL to tune the gap dependence of first field integrals of the phase shifters to very low tolerances [9-11].

\section{A. Description of the method}

As demonstrated in Fig. 4, one can tune pole deformation to a symmetric balance between concave at small and convex at large gaps. However, total deformation cannot be reduced in this way. If it is too large, the phase jitter gets out of the specification window as shown in Fig. 7(b). Using suitable shims, however, is an effective method to induce targeted gap-dependent modifications of the magnetic field distribution.

Shims made of 0.1-0.4 mm soft iron foil are used, which have the same dimensions as the magnet surface [12-15]. If placed on a magnet in between two poles, the field of these two poles is weakened as sketched in Fig. 8. For symmetry reasons, there is no net steering. This leads to a $K$ parameter change of $\Delta K$ resulting in a phase jump of

$$
\Delta \varphi=2 \pi \frac{K \cdot \Delta K}{1+0.5 K^{2}}
$$

For shims $\Delta K$ is always negative and $\Delta \varphi$ is negative as well.

Alternatively, a phase "jump" $\Delta \varphi$ can also be generated by pole tuning: By symmetrically changing the local gap of a pair of neighboring poles, the sign of $\Delta K$ can be chosen and the strength can be varied continuously by increasing or decreasing the local gap. This is a marked difference to shimming.

The gap dependencies of shims and pole tuning were investigated experimentally. The results are presented in Fig. 9. Measurements were done by using the U40-X014 again. On one period, the pole height of two poles was adjusted symmetrically by $-0.05,+0.025,+0.05$, and $+0.1 \mathrm{~mm}$. On another period sufficiently distant away, full magnet shims of $0.1,0.2,0.3$, and $0.4 \mathrm{~mm}$ thickness were applied. The phase jump was measured for the operational gap range from 10 to $20 \mathrm{~mm}$ in $2 \mathrm{~mm}$ steps. The results are shown in Fig. 9(a). The abscissa shows the amount of pole height tuning or the shim thickness, respectively. The amount of phase jump is given by the ordinate. For pole height tuning shown in the left upper part of Fig. 9(a), it is observed that the phase jumps are proportional to the amount of tuning, but all for all gaps the jumps are identical. There is no gap dependence. Also, positive and negative jumps are possible. For the shims, the situation is different: There is significant gap dependence, and all jumps are negative.

In addition, Fig. 9(a) gives a good impression of the linear dependence on small pole adjustments or small shim 

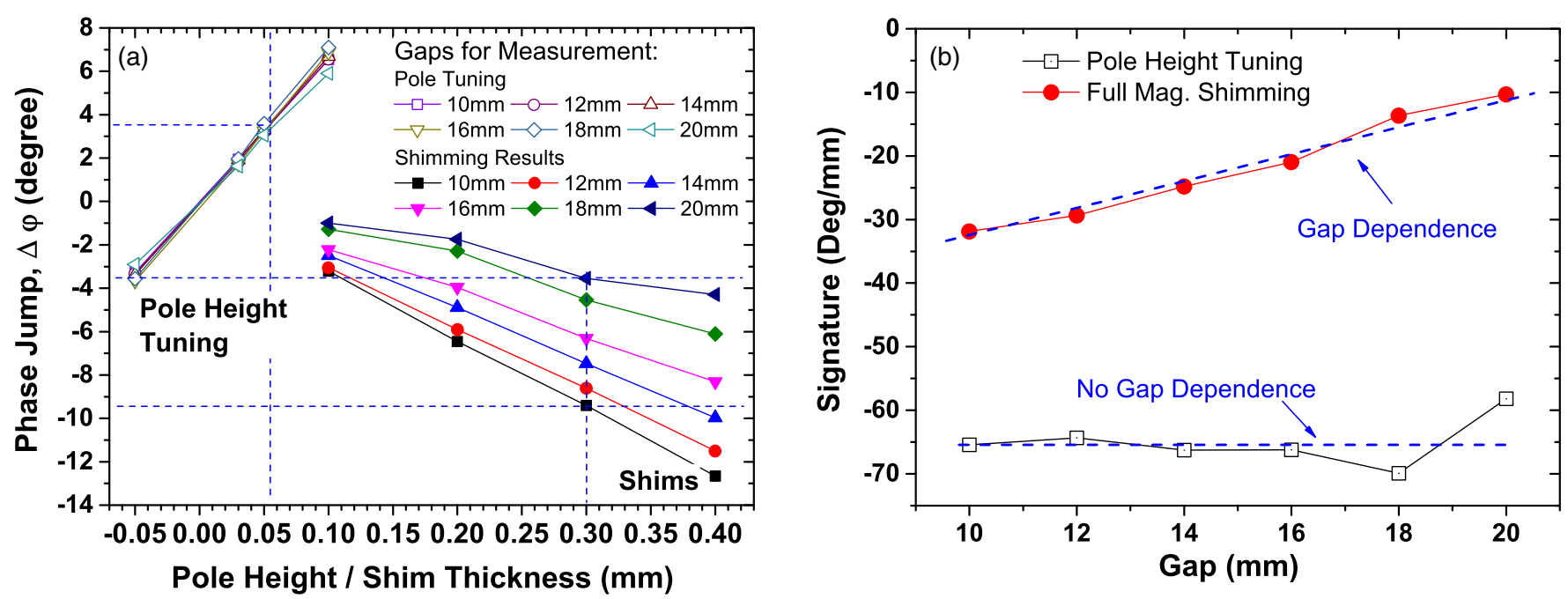

FIG. 9. (a) Gap dependence of phase jumps $\Delta \boldsymbol{\varphi}$ induced by tuning the height of a pair of poles and by shims of different thicknesses. The abscissa shows the amount of pole tuning or shim thickness. The dashed lines should guide the eye for the determination the shimming strengths; see the text. (b) Resulting signatures. For pole height tuning, there is no gap dependence. Note that for direct quantitative comparison the sign of the signature of pole height tuning was reversed. The undulator X014 is used for the measurement.

thicknesses, which is an important assumption for both methods $[6,7,12,13]$.

Phase jumps normalized to pole shifts or shim thicknesses are called signatures and are shown in Fig. 9(b). Note that for the sake of direct comparison the sign of the signature for pole height tuning was reversed. These signatures are the basis for gap-dependent phase tuning. It is obvious that shims and pole height tuning have different gap dependencies. This effect is used for compensation.

The gap independence of the phase jump by pole height tuning is a quite surprising experimental fact. It was

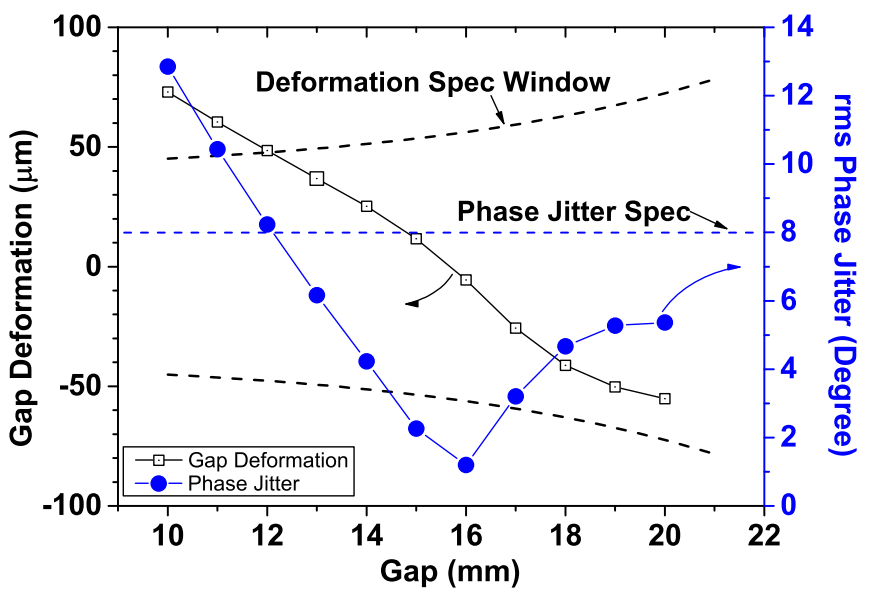

FIG. 10. Effect of shifting the tuning gap to $16 \mathrm{~mm}$ on gap deformation (open squares) and the rms phase jitter (full circles) as a function of the undulator gap. Now at $20 \mathrm{~mm}$ specifications are fulfilled but exceeded at small gaps. investigated and can be explained by the local gap dependence of $\Delta K$ of a pair of neighboring poles. They behave different than the change in $K$ expected using Eqs. (2) and (4), which applies to a periodic structure. However, gap independence is not essential for the compensation method described below. The difference in gap dependencies is important.

\section{B. Phase tuning example}

The X014 is used as the example to demonstrate the tuning strategy. The original gap deformation and phase jitter after the standard EXFEL tuning procedure using a tuning gap of $14 \mathrm{~mm}$ was already illustrated in Fig. 7: The gap deformation and phase jitter at small gaps are close to the limits and in large gaps well above.

In a first step, poles were retuned so that at $20 \mathrm{~mm}$ gap the phase jitter is well within specs. This is done by shifting the tuning gap to $16 \mathrm{~mm}$, where now the phase jitter is $1.19^{\circ}$ only; see Fig. 10. This is the main work, since it requires one to retune slightly all 248 poles of the undulator. Now at 10 and $20 \mathrm{~mm} 12.85^{\circ}$ and $5.36^{\circ}$ are obtained, respectively. Since pole tuning cannot reduce total deformation, only the gap range of the phase error was shifted. Now the low gap region is out of specs as seen in Fig. 10.

Next, the $K$ parameter for phase error calculation is slightly reduced, resulting in an additional error; see Fig. 11, black and red curve. The $K$ parameter is chosen such that the phase error at the beginning and end is about the same. For this purpose the $K$ parameter was reduced slightly by 0.0035 or $0.089 \%$ from 3.9009 to 3.8974 . This initially increases the total phase error. But now there is a long section from $z \approx-1300$ to $1300 \mathrm{~mm}$ where the phase 


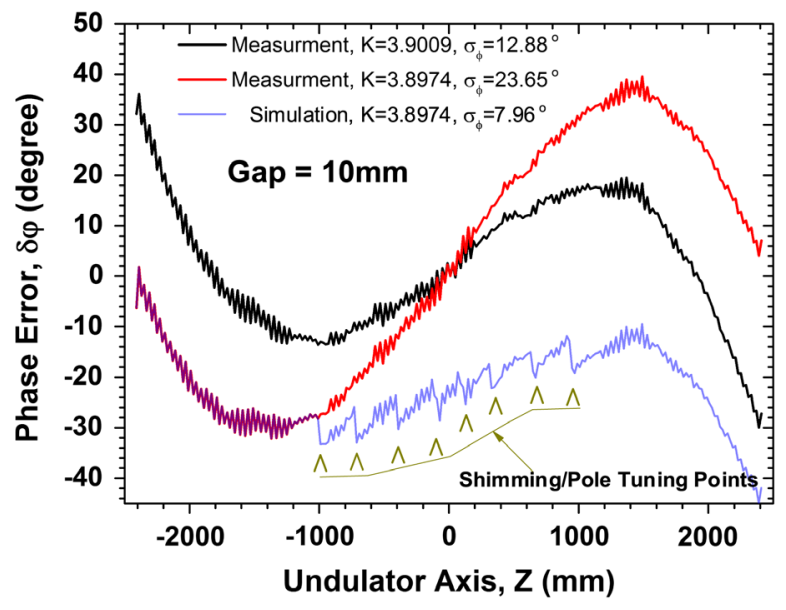

FIG. 11. Phase error $\delta \varphi$ at $10 \mathrm{~mm}$ gap. Black: $K=3.9009$, resulting in a minimum rms phase jitter of $12.88^{\circ}$. Red curve: $K=3.8974$. Phase error at the start and end are approximately the same. Blue: Expected phase error with $K=3.8974$ after shimming. The expected rms error is $7.95^{\circ}$.

error increases almost linearly with positive slope and the end sections with negative slope are shorter.

In the linear section, phase shims are placed, each reducing the phase error by a step amount. Parameters were selected by the following consideration using Figs. 11 and 9(a): a) In Fig. 11, the positive increase of phase errors in the center region extends from $-30^{\circ}$ to $+40^{\circ}$. Only about $50^{\circ}$ should be compensated by shims in order to limit the impact of the outer end sections with negative slope on the rms phase jitter. However, the properties at $20 \mathrm{~mm}$ must stay unaffected. b) In order to do so at that gap, the effect of phase shims and pole height tuning must cancel mutually. Using the dashed lines as guides for the eye in Fig. 9(a), it is seen that a $0.3 \mathrm{~mm}$ shim

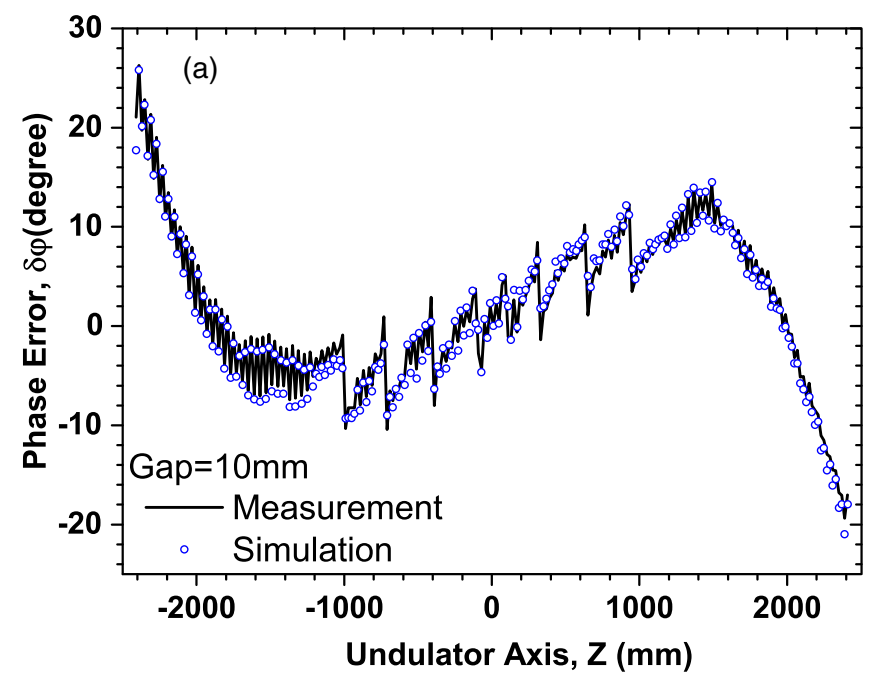

at $20 \mathrm{~mm}$ creates a negative phase jump of $-3.5^{\circ}$ which needs to be compensated by a pole shift of $+0.055 \mathrm{~mm}$, which creates $+3.5^{\circ}$. At $10 \mathrm{~mm}$ this shim creates a phase jump of $-9.5^{\circ}$, while the pole contribution is constant at $3.5^{\circ}$. The net effect is $-6^{\circ}$ per shim or pole pair, and consequently eight corrections are needed to compensate $+48^{\circ}$ and approximately fulfill the requirement. The effect of applying these corrections was simulated and is shown by the blue curve in Fig. 11. The rms simulated phase jitter is $7.95^{\circ}$ and inside EXFEL specs.

The final results for the optical phase are shown in Fig. 12(a). The black line shows the measured phase error $\delta \varphi$ at the gap of $10 \mathrm{~mm}$. The blue circles show the simulated results shown in Fig. 11 shifted up by $24^{\circ}$ to match the same scale. There is very good agreement. Finally, Fig. 12(b) shows the measured phase jitter as a function of the gap. It demonstrates that the rms phase jitter at the gap of $10 \mathrm{~mm}$ is reduced to $7.65^{\circ}$, as expected and within specs at all gaps.

\section{SUMMARY AND OUTLOOK}

Gap-dependent parabolic girder deformation is commonly observed on the undulators for the EXFEL and is found to be the dominant source for the rms phase jitter. Analytical formulas are developed which allow a quantitative evaluation and the definition of specifications. Results can be universally applied.

Considerable variation of girder deformation has been observed throughout the undulators built for the EXFEL. While most are well within specs, some come close, but only one out of 91 was found to be out of specs, and girder deformation could not be tolerated.

For mitigation a systematic method using shims was developed and is presented in detail. It makes use of the

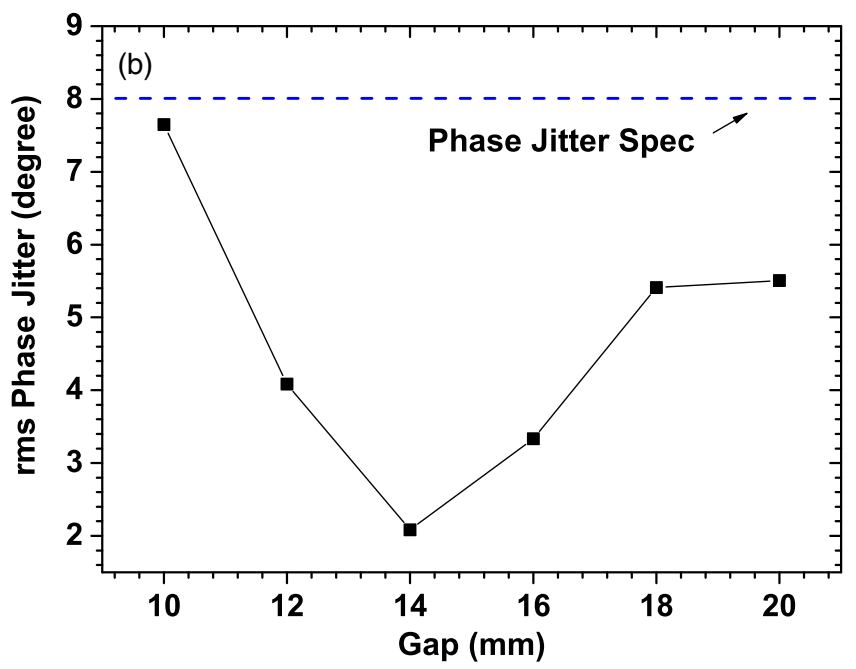

FIG. 12. (a) Black curve: Measured phase error $\delta \varphi$ after tuning. Blue open circles: Simulation of Fig. 11 shifted by $24^{\circ}$ to match scales. (b) Final gap dependence of the rms phase jitter as a function of the undulator gap. 
different gap dependencies of full magnet shims and pole height tuning as used for tuning of the EXFEL devices. Measured gap signatures for both cases are used. With this method the rms phase jitter observed initially on one device, which did not comply with specs, could be reduced by about $5^{\circ}$ with very moderate effort and brought back to specifications.

In general, the girder deformation length compare to the undulator period is much longer. Therefore, its effect on field integral error is limited. By using the method described in this report, the negative effects resulting from small gap-dependent girder deformation of $110 \mu \mathrm{m}$ can be fully compensated without negative side effects: The transverse overlap between the electron beam and laser field is guaranteed by specs on the first and second field integrals, and they are not affected by the tuning at all.

The longitudinal overlap between the microbunched electron beam and the laser field is preserved if the specs on phase jitter are complied. The local peak field variation leads to an according $K$ parameter and phase variation along the undulator as seen in Fig. 5. But exactly this gap dependency is counteracted and brought back to specs.

It can thus be concluded that for the observed girder deformation of about $110 \mu \mathrm{m}$ all negative effects on magnetic specifications can be well compensated with this method.

A potential application of the method, which would be straightforward to realize, could be to significantly reduce the gap dependence of the phase jitter well below the specs given in the paper. This was not needed for EXFEL but might be of great use for long undulators in a storage ring operated on high harmonics. For the EXFEL devices, a phase jitter of $2^{\circ}$ or less over the whole operational gap range seems feasible. Alternatively, the girder stiffness could be reduced by tolerating more mechanical gap deformation. Thus, a trade-off between mechanical effort and a moderate increase of magnetic measurements and tuning can be obtained.

\section{ACKNOWLEDGMENTS}

The authors gratefully acknowledge the technical support by K. Berndgen, M. Bagha-Shanjani, and M. Knoll.

[1] M. Altarelli et al., technical design report, ISBN 3-93570217-5, 2006; http://www.xfel.eu/dokumente/technical_ documents.

[2] A. M. Kondratenko and E. L. Saldin, Part. Accel. 10, 207 (1980).

[3] R. Bonifacio, C. Pellegrini, and L. M. Narducci, Opt. Commun. 50, 373 (1984).

[4] P. Elleaume, J. Chavanne, and B. Faatz, Nucl. Instrum. Methods Phys. Res., Sect. A 455, 503 (2000).

[5] Y. Li, B. Faatz, and J. Pflueger, Phys. Rev. ST-AB 11, 100701 (2008).

[6] J. Pflüger, H. Lu, and T. Teichmann, Nucl. Instrum. Methods Phys. Res., Sect. A 429, 386 (1999).

[7] Y. Li and J. Pflueger (to be published).

[8] J. Pflueger et al., in TUPSO60, Proceedings of the FEL2013, New York, 2014.

[9] Y. Li and J. Pflueger, Phys. Rev. ST-AB 18, 030703 (2015).

[10] Y. Li and J. Pflueger, internal EXFEL Report No. WP71/ 2012/15 (unpublished).

[11] Y. Yang, Y. Li, H. Lu, F. Wolff-Fabris, and J. Pflueger et al., in TUPSO60, Proceedings of the FEL2013, New York, 2014.

[12] J. M. Slater, S. C. Gottschalk, F. E. James, D. C. Quimby, K. E. Robinson, and A. S. Valla, Rev. Sci. Instrum. 60, 1881 (1989).

[13] D. C. Quimby, S. C. Gottschalk, F. E. James, K. E. Robinson, J. M. Slater, and A. S. Valla, Nucl. Instrum. Methods Phys. Res., Sect. A 285, 281 (1989).

[14] J. Chavanne, E. Chinchio, M. Diot, P. Elleaume, D. Frachon, X. Marechal, C. Mariaggi, and F. Revol, Rev. Sci. Instrum. 63, 317 (1992).

[15] J. Chavanne and P. Elleaume, in Undulators and Wigglers and their Applications (Taylor \& Francis, London, 2003), Chap. 5. 